\title{
The continuum parabolic Anderson model with a half-Laplacian and periodic noise
}

\author{
Alexander Dunlap*
}

\begin{abstract}
We construct solutions of a renormalized continuum fractional parabolic Anderson model, formally given by $\partial_{t} u=-(-\Delta)^{\frac{1}{2}} u+\xi u$, where $\xi$ is a periodic spatial white noise. To be precise, we construct limits as $\varepsilon \rightarrow 0$ of solutions of $\partial_{t} u_{\varepsilon}=-(-\Delta)^{\frac{1}{2}} u_{\varepsilon}+$ $\left(\xi_{\varepsilon}-C_{\varepsilon}\right) u_{\varepsilon}$, where $\xi_{\varepsilon}$ is a mollification of $\xi$ at scale $\varepsilon$ and $C_{\varepsilon}$ is a logarithmically diverging renormalization constant. We use a simple renormalization scheme based on that of Hairer and Labbé, "A simple construction of the continuum parabolic Anderson model on $\mathbf{R}^{2}$."
\end{abstract}

Keywords: parabolic Anderson model; fractional Laplacian.

MSC2020 subject classifications: $60 \mathrm{H} 15$.

Submitted to ECP on March 13, 2020, final version accepted on August 12, 2020.

\section{Introduction}

Let $\Lambda=-(-\Delta)^{\frac{1}{2}}$ be the half-Laplacian on $\mathbf{R}$. It is given by the formula

$$
\Lambda f(x)=\frac{1}{\pi} \mathrm{p} \cdot \mathrm{v} \cdot \int_{\mathbf{R}} \frac{f(y)-f(x)}{(y-x)^{2}} \mathrm{~d} y .
$$

Here and throughout the paper, p.v. $\int_{\mathbf{R}}$ will denote the principal value integral: if $g$ is a function with a singularity at $x$, then

$$
\text { p.v. } \int_{\mathbf{R}} g(y) \mathrm{d} y=\lim _{\varepsilon \downarrow 0} \int_{\mathbf{R} \backslash[x-\varepsilon, x+\varepsilon]} g(y) \mathrm{d} y .
$$

Also, let $\xi$ be a periodic Gaussian spatial white noise on $\mathbf{R}$ of period $L \in(0, \infty)$. The covariance kernel of $\xi$ is thus given by $\mathbf{E} \xi(x) \xi(y)=\sum_{k \in \mathbf{Z}} \delta(x-y+k L)$. We are interested in the fractional parabolic Anderson model (PAM) formally given by

$$
\partial_{t} u=\Lambda u+\xi u ; \quad u(0, \cdot)=\underline{u} .
$$

We would expect solutions of (1.1) to model scaling limits of a PAM on the lattice with long-range jumps. This lattice model, with a non-Gaussian noise, was previously studied in [15]. We refer, for example, to [13] for more background on the PAM.

Straightforward heuristics indicate that (1.1) cannot be interpreted directly. Indeed, the white noise $\xi$ has (Hölder) regularity “ $-\frac{1}{2}-$ " (i.e. any regularity strictly below $-\frac{1}{2}$ ),

\footnotetext{
*Department of Mathematics, Stanford University, Stanford, CA 94305, USA. E-mail: aj dunl2@stanford . edu
} 
and thus the solution to the linearized problem (around $u \equiv 1$ ) of (1.1) has regularity $\frac{1}{2}-$, since we gain one derivative by inverting the half-Laplacian. So we can expect the regularity of $u$ to be at most $\frac{1}{2}-$. Thus the product of $\xi$ and $u$ is undefined since the sum of their regularities is (just barely) negative. This is why the power of $\frac{1}{2}$ on the Laplacian is interesting-it is the largest power such that the product in (1.1) is ill-defined.

Since abstract theory does not allow us to interpret the problem as stated, we turn our attention to a regularized problem and try to pass to a limit as the regularization is removed. We will see that a renormalization is necessary to obtain a finite limit. Fix a mollifier $\rho \in \mathcal{C}_{\mathrm{c}}^{\infty}$ (i.e. smooth with compact support) so that $\int_{\mathbf{R}} \rho \equiv 1$. For $\varepsilon>0$, define $\rho_{\varepsilon}(x)=\varepsilon^{-1} \rho\left(\varepsilon^{-1} x\right)$ and $\xi_{\varepsilon}=\rho_{\varepsilon} * \xi$, where $*$ denotes spatial convolution. Fix a constant $C_{\varepsilon} \in \mathbf{R}$, depending on $\varepsilon$, and an initial condition $\underline{u}$. Then we consider the problem

$$
\partial_{t} u_{\varepsilon}=\Lambda u_{\varepsilon}+\left(\xi_{\varepsilon}-C_{\varepsilon}\right) u_{\varepsilon} ; \quad u_{\varepsilon}(0, \cdot)=\underline{u} .
$$

This equation can be solved using standard techniques because $\xi_{\varepsilon} \in \mathcal{C}^{\infty}$ for all $\varepsilon>0$. Our goal will be to pass to the limit as $\varepsilon \rightarrow 0$. To state our main theorem, we first define the Banach space in which this convergence takes place. If $\mathcal{Y}$ is a Banach space, we define for $\kappa \in \mathbf{R}$ and $T>0$ the Banach space $\mathcal{X}_{T}^{\kappa}(\mathcal{Y})$ to be the space of functions $f \in \mathcal{C}_{\text {loc }}((0, T] ; \mathcal{Y})$ with finite norm

$$
\|f\|_{\mathcal{X}_{T}^{\kappa}(\mathcal{Y})}:=\sup _{t \in(0, T]} t^{1-\kappa}\|f(t, \cdot)\| \mathcal{Y} .
$$

Theorem 1.1. There is a choice of deterministic constants $C_{\varepsilon}, \varepsilon \in(0,1]$ (explicitly defined in (5.1) below), so that the following holds. For any $\kappa \in(0,1 / 4)$, if $\underline{u} \in \mathcal{C}^{-\frac{1}{2}+2 \kappa}$, then for each $\varepsilon \in[0,1]$ there is a random $u_{\varepsilon} \in \mathcal{C}_{\text {loc }}\left((0, \infty) ; \mathcal{C}^{\frac{1}{2}-\kappa}\right)$ so that whenever $\varepsilon>0$, $u_{\varepsilon}$ is a mild solution to (1.2), and moreover for every $T>0, u_{\varepsilon} \rightarrow u_{0}$ in probability in $\mathcal{X}_{T}^{\kappa}\left(\mathcal{C}^{\frac{1}{2}-\kappa}\right)$. Finally, we have a constant $C<\infty$ so that, for all $\varepsilon \in(0,1]$,

$$
\left|C_{\varepsilon}-(1 / \pi) \log (1 / \varepsilon)\right| \leq C .
$$

The model (1.1) has similar local scaling properties to the continuum PAM

$$
\partial_{t} u=\Delta u+\xi u
$$

in two spatial dimensions. That model also has a just-barely-ill-defined product, and it also requires a logarithmic renormalization. Solutions to (1.5) on a compact domain were constructed independently in $[10,9]$ using the theories of regularity structures and paracontrolled distributions, respectively. An elementary approach that also works on the whole space was carried out by Hairer and Labbé in [11], and some properties of solutions were derived in [8,7]. The more difficult case of (1.5) in three spatial dimensions was tackled in [12]. On the other hand, singular stochastic PDEs involving fractional Laplacian terms have previously been considered in [2, 3].

Our approach to proving Theorem 1.1 closely follows the strategy of [11], avoiding the use of regularity structures or paracontrolled distributions. Similar strategies were used for the random Schrödinger equation in [5, 4]. As in [11], we perform a change of variables in (1.2) by writing $u_{\varepsilon}=\mathrm{e}^{S_{\varepsilon}} v_{\varepsilon}$, where $S_{\varepsilon}$ is an approximate solution to the linearized time-independent problem, and write a PDE for $v_{\varepsilon}$. (See Section 3.) The coefficients of the PDE for $v_{\varepsilon}$ converge, in appropriate spaces, as $\varepsilon \downarrow 0$. One of these converging "coefficients" is in fact a nonlocal operator. Proving the convergence requires new estimates, which we carry out in Section 5 using some purely analytic bounds that we prove in Section 4 . Then the continuity of the PDE for $v_{\varepsilon}$ shows that $v_{\varepsilon} \rightarrow v$, where $v$ solves the limiting PDE. This is the main content of Section 6 and is essentially the same as the argument of [11], as the estimates having been obtained by this point are 
analogous. It is also easy to see that $S_{\varepsilon}$ converges to a limit $S$ as $\varepsilon \downarrow 0$. Inverting the change of variables then shows that $u_{\varepsilon}$ converges to $\mathrm{e}^{-S} v$.

In this paper, we restrict ourselves to the case of periodic noise. The periodicity is used so that the noise is bounded (as a distribution in $\mathcal{C}^{-\frac{1}{2}-\kappa}$ for any $\kappa>0$ ) uniformly in space. It is not clear whether or how solutions to (1.1) can be constructed with aperiodic white noise. In particular, the weighted-space approach of [11] does not immediately generalize to our setting, because the Cauchy kernel decays only algebraically in space, in contrast to the Gaussian decay of the heat kernel.

\section{Preliminaries and notation}

We will often work with constants, which we call $C$, and allow them to change from line to line in a computation. This does not apply to the renormalization constant $C_{\varepsilon}$, which will be fixed in (5.1) below.

\subsection{Hölder spaces}

We will work in $\alpha$-Hölder spaces, given as usual by the norm

$$
\|u\|_{\mathcal{C}^{\alpha}}=\|u\|_{L^{\infty}}+\sup _{|x-y| \leq 1} \frac{|u(x)-u(y)|}{|x-y|^{\alpha}}
$$

for all $\alpha \in(0,1)$. We will also use Hölder spaces with negative Hölder exponent. Put

$$
\eta_{x}^{\lambda}(y)=\lambda^{-1} \eta\left(\lambda^{-1}(y-x)\right)
$$

for any function $\eta$. Then, for all $\alpha \in(-1,0)$, the $\alpha$-Hölder norm of a distribution $u$ is

$$
\|u\|_{\mathcal{C}^{\alpha}}=\sup \left\{\lambda^{-\alpha}\left|u\left(\eta_{x}^{\lambda}\right)\right|: x \in \mathbf{R}, \eta \in \mathcal{C}^{1}([-1,1]),\|\eta\|_{\mathcal{C}^{0}}=1, \lambda \in(0,1]\right\} .
$$

Let $\mathcal{C}^{\alpha}$ be the Banach space of distributions such that this norm is finite. We recall that $\mathcal{C}^{\alpha}$ is equivalent to the Besov space $\mathcal{B}_{\infty, \infty}^{\alpha}$ (see [1]), and refer to [11, Section 2], [10, Section 3], or [6] for background on the use of negative Hölder spaces for stochastic PDEs. We will use the following wavelet characterization of negative Hölder spaces.

Proposition 2.1 ([11, Proposition 2.4] or [6, Definition 2.8 and Proposition 2.14]). There are compactly-supported functions $\psi, \phi \in \mathcal{C}_{\mathrm{c}}^{1}$ so that for any $\alpha \in(-1,0)$ we have a constant $C<\infty$ so that (using the notation (2.1))

$$
\|f\|_{\mathcal{C}^{\alpha}} \leq C \sup _{x \in \mathbf{Z}}\left(\left|\int f \phi_{x}^{1}\right|+\sup _{n \in \mathbf{N}} 2^{\alpha n}\left|\int_{\mathbf{R}} f \psi_{2^{-n} x}^{2^{-n}}\right|\right) .
$$

The following statement about multiplication of elements of Hölder spaces is standard.

Lemma 2.2 ([1, Theorem 2.52]). If $\alpha<\beta$ and $\alpha+\beta>0$, then multiplication of functions extends to a continuous bilinear map $\mathcal{C}^{\alpha} \times \mathcal{C}^{\beta} \rightarrow \mathcal{C}^{\alpha}$.

\subsection{The fractional Laplacian}

In this section we establish some necessary background results on the fractional Laplacian, especially on inverting the fractional Laplacian $\Lambda$ and the fractional heat operator $\partial_{t}-\Lambda$. We recall (see e.g. [14]) the equivalent definition

$$
\Lambda f(x)=\frac{1}{\pi} \int_{\mathbf{R}} \frac{f(y)-f(x)-(y-x) f^{\prime}(x) \mathbf{1}\{|y-x| \leq 1\}}{(y-x)^{2}} \mathrm{~d} y .
$$

The regularization $(y-x) f^{\prime}(x) \mathbf{1}\{|y-x| \leq 1\}$ obviates the need for the principal value.

We let $\delta$ denote a Dirac delta distribution at 0 . We will work with an approximate Green's function of the fractional Laplacian, defined in the following lemma. 
Lemma 2.3. There is a smooth even function $G: \mathbf{R} \backslash\{0\} \rightarrow \mathbf{R}$ so that $\operatorname{supp} G \subset[-1,1]$,

$$
G(x)=(1 / \pi) \log |x| \text { for all } x \in[-1 / 2,1 / 2]
$$

and if $F=\Lambda G-\delta$, then $F$ is smooth and there is a constant $C$ so that, for all $x \in \mathbf{R}$,

$$
|F(x)|,\left|F^{\prime}(x)\right| \leq C(1+|x|)^{-2} .
$$

Proof. Let $\widetilde{G}(x)=\frac{1}{\pi} \log |x|$ for all $x \in \mathbf{R} \backslash\{0\}$. It is standard that $\Lambda \widetilde{G}=\delta$ in the sense of distributions. Take $G$ to be any smooth even function $\mathbf{R} \backslash\{0\} \rightarrow \mathbf{R}$ such that $\operatorname{supp} G \subset[-1,1]$ and $\left.G\right|_{\left[-\frac{1}{2}, \frac{1}{2}\right]}=\left.\widetilde{G}\right|_{\left[-\frac{1}{2}, \frac{1}{2}\right]}$. Define $F=\Lambda G-\delta=\Lambda(G-\widetilde{G})$. Since $G-\widetilde{G}$ is smooth, $F$ is smooth as well. The estimate (2.4) is then an easy consequence of the decay of the kernel in (2.2).

We also will need a Schauder-type estimate for $G$.

Lemma 2.4. If $\alpha \in(-1,0)$, there is a $C<\infty$ so that if $f \in \mathcal{C}^{\alpha}$ then $G * f \in \mathcal{C}^{\alpha+1}$ and $\|G * f\|_{\mathcal{C}^{\alpha+1}} \leq C\|f\|_{\mathcal{C}^{\alpha}}$.

Proof. If $\eta$ is a smooth, positive function, supported on $\left[-\frac{1}{2}, \frac{1}{2}\right]$, identically 1 in a neighborhood of 0 , then

$$
L(x):=\int_{0}^{1}(1 / y) \eta(x / y) \mathrm{d} y=\int_{0}^{1 / x}(1 / y) \eta(1 / y) \mathrm{d} y=\pi G(x)+k(x)
$$

for some smooth, compactly-supported function $k$. Then it is sufficient to prove that $\|L * f\|_{\mathcal{C}^{\alpha+1}} \leq C\|f\|_{\mathcal{C}^{\alpha}}$. To do this, we note first that $(L * f)(x)=\int_{0}^{1}\left(\frac{1}{y} \eta(\dot{\bar{y}}) * f\right)(x) \mathrm{d} y$. Fix $x<x^{\prime}$ and note that

$$
(L * f)(x)-(L * f)\left(x^{\prime}\right)=\int_{0}^{1} \int \frac{1}{y} q_{x, x^{\prime}, y}\left(\frac{z}{y}\right) f(z) \mathrm{d} z \mathrm{~d} y,
$$

where $q_{x, x^{\prime}, y}(z)=\eta(x / y-z)-\eta\left(x^{\prime} / y-z\right)$ and we use the common abuse of notation in which the integrals in $z$ are in fact pairings with the distribution $f$. If $y \geq 2\left|x-x^{\prime}\right|$, then $\left\|q_{x, x^{\prime}, y}\right\|_{\mathcal{C}^{0}} \leq\|\eta\|_{\mathcal{C}^{1}} \frac{\left|x-x^{\prime}\right|}{y}$ and $\operatorname{supp} q_{x, x^{\prime}, y}$ is contained in an interval of width 2 , so $\left|\int \frac{1}{y} q_{x, x^{\prime}, y}(z / y) f(z) \mathrm{d} z\right| \leq\|\eta\|_{\mathcal{C}^{1}}\|f\|_{\mathcal{C}^{\alpha}}\left|x-x^{\prime}\right| y^{\alpha-1}$. On the other hand, if $0<y \leq 2\left|x-x^{\prime}\right|$, then $q_{x, x^{\prime}, y}$ can be written as the sum of two $\mathcal{C}^{1}$ functions with support contained in $\left[-\frac{1}{2}, \frac{1}{2}\right]$, each with $\mathcal{C}^{0}$ norm $\|\eta\|_{\mathcal{C}^{0}}$, so $\left|\int \frac{1}{y} q_{x, x^{\prime}, y}\left(\frac{z}{y}\right) f(z) \mathrm{d} z\right| \leq 2\|\eta\|_{\mathcal{C}^{0}}\|f\|_{\mathcal{C}^{\alpha}} y^{\alpha}$. Therefore, we have, for a constant $C$ depending on $\eta$ but not on $f$, that

$$
\begin{aligned}
\left|(L * f)(x)-(L * f)\left(x^{\prime}\right)\right| & \leq C\left(\int_{0}^{2\left|x-x^{\prime}\right|} y^{\alpha} \mathrm{d} y+\left|x-x^{\prime}\right| \int_{2\left|x-x^{\prime}\right|}^{1} y^{\alpha-1} \mathrm{~d} y\right)\|f\|_{\mathcal{C}^{\alpha}} \\
& \leq C\left|x-x^{\prime}\right|^{\alpha+1}\|f\|_{\mathcal{C}^{\alpha}}
\end{aligned}
$$

The necessary bound on $|(L * f)(x)|$ is easier, so we omit it.

The inverse of the fractional heat operator $\partial_{t}-\Lambda$ is the Cauchy kernel $P_{t}(x)=\frac{t}{\pi\left(t^{2}+x^{2}\right)}$. We will need the following Schauder-type estimate for this kernel.

Lemma 2.5. For any $T<\infty$ and $\alpha<\beta$, there is a $C<\infty$ so that for any function $f \in \mathcal{C}^{\alpha}$ and any $t \in(0, T]$, we have $P_{t} * f \in \mathcal{C}^{\infty}$ and $\left\|P_{t} * f\right\|_{\mathcal{C}^{\beta}} \leq C t^{-(\beta-\alpha)}\|f\|_{\mathcal{C}^{\alpha}}$. 
Proof. This follows from a scaling argument analogous to that used in [11, Lemma 2.8]. For completeness, we present the argument for the case $-1<\alpha<0<\beta<1$, which is what we use. As in [10, Lemma 5.5], ${ }^{1}$ fix a smooth function $\omega:[0, \infty) \rightarrow[0, \infty)$ so that supp $\omega \subset\left[\frac{1}{2}, 2\right]$ and $\sum_{n \in \mathbf{Z}} \omega\left(2^{n} \cdot\right) \equiv 1$. Then define, for $t \geq 0$ and $x \in \mathbf{R}, P_{t}^{(n)}(x):=$ $\omega\left(2^{n}(t+|x|)\right) P_{t}(x)$, so we have $\sum_{n \in \mathbf{Z}} P_{t}^{(n)}=P_{t}$ and (since $\left.P_{t}(x)=2^{n} P_{2^{n} t}\left(2^{n} x\right)\right) P_{t}^{(n)}(x)=$ $2^{n} P_{2^{n} t}^{(0)}\left(2^{n} x\right)$. Define $P_{t}^{-}=\sum_{n<0} P_{t}^{(n)}$ and $P_{t}^{+}=\sum_{n \geq 0} P_{t}^{(n)}$. We note that there is a constant $C<\infty$ so that for all $t \in(0, T]$, we have the estimate $\left|P_{t}^{-}(x)\right|,\left|\partial_{x} P_{t}^{-}(x)\right| \leq C(1+|x|)^{-2}$ for all $x \in \mathbf{R}$. This implies that $\left\|P_{t}^{-} * f\right\|_{\mathcal{C}^{\beta}} \leq C\|f\|_{\mathcal{C}^{\alpha}}$. On the other hand, we have $\left\|P_{t}^{(0)}\right\|_{\mathcal{C}^{2}} \leq C$ for all $t \in(0, T]$. Therefore, we have that $\left\|2^{n} P_{t}^{(0)}\left(2^{n} \cdot\right) * f\right\|_{\mathcal{C}^{0}} \leq C 2^{-n \alpha}\|f\|_{\mathcal{C}^{\alpha}}$ and $\left\|\partial_{x}\left[2^{n} P_{t}^{(0)}\left(2^{n} \cdot\right)\right] * f\right\|_{\mathcal{C}^{0}} \leq C 2^{n(1-\alpha)}\|f\|_{\mathcal{C}^{\alpha}}$, so

$$
\left\|2^{n} P_{t}^{(0)}\left(2^{n} \cdot\right) * f\right\|_{\mathcal{C}^{\beta}} \leq C\left\|2^{n} P_{t}^{(0)}\left(2^{n} \cdot\right) * f\right\|_{\mathcal{C}^{0}}^{1-\beta}\left\|2^{n} P_{t}^{(0)}\left(2^{n} \cdot\right) * f\right\|_{\mathcal{C}^{1}}^{\beta} \leq C 2^{n(\beta-\alpha)}\|f\|_{\mathcal{C}^{\alpha}} .
$$

We now complete the proof by concluding that

$$
\left\|P_{t}^{+} * f\right\|_{\mathcal{C}^{\beta}} \leq C \sum_{n=0}^{\left\lceil-\log _{2} t\right\rceil+1}\left\|2^{n} P_{2^{n} t}^{(0)}\left(2^{n} \cdot\right) * f\right\|_{\mathcal{C}^{\beta}} \leq C\|f\|_{\mathcal{C}^{\alpha}} \sum_{n=0}^{\left\lceil-\log _{2} t\right\rceil+1} 2^{n(\beta-\alpha)} \leq \frac{C\|f\|_{\mathcal{C}^{\alpha}}}{t^{\beta-\alpha}}
$$

\section{The change of variables}

In this section we explain the key change of variables that we perform on (1.2). This change of variables is an analogue for the fractional Laplacian of the change of variables performed in [11, p. 3]. The advantage of the change of variables is that the coefficients of the new equation converge as $\varepsilon \downarrow 0$, and so an equation is obtained for the limit.

Lemma 3.1. For $\varepsilon \geq 0$, let $S_{\varepsilon}=-G * \xi_{\varepsilon}$, where $G$ is defined as in Lemma 2.3. For $\varepsilon>0$, if we put $u_{\varepsilon}=\mathrm{e}^{S_{\varepsilon}} v_{\varepsilon}$, then $v_{\varepsilon}$ satisfies

$$
\partial_{t} v_{\varepsilon}=\Lambda v_{\varepsilon}+v_{\varepsilon}\left[-F * \xi_{\varepsilon}+Z_{\varepsilon}\right]+\Xi_{\varepsilon} v_{\varepsilon}, \quad v_{\varepsilon}(0, \cdot)=\mathrm{e}^{-S_{\varepsilon}} \underline{u},
$$

where

$$
\begin{gathered}
Z_{\varepsilon}(x)=\tilde{Z}_{\varepsilon}(x)-C_{\varepsilon}, \quad \tilde{Z}_{\varepsilon}(x)=\frac{1}{\pi} \mathrm{p} \cdot \mathrm{v} \cdot \int \frac{\mathrm{e}^{S_{\varepsilon}(y)-S_{\varepsilon}(x)}-\left(1+S_{\varepsilon}(y)-S_{\varepsilon}(x)\right)}{(y-x)^{2}} \mathrm{~d} y, \\
\Xi_{\varepsilon} w(x)=\frac{1}{\pi} \mathrm{p} \cdot \mathrm{v} \cdot \int \frac{\left(\mathrm{e}^{S_{\varepsilon}(y)-S_{\varepsilon}(x)}-1\right)(w(y)-w(x))}{(y-x)^{2}} \mathrm{~d} y .
\end{gathered}
$$

Proof. The initial condition is clear, so it remains to verify the PDE. We note that

$$
\partial_{t} v_{\varepsilon}=\mathrm{e}^{-S_{\varepsilon}} \partial_{t} u_{\varepsilon}=\mathrm{e}^{-S_{\varepsilon}}\left[\Lambda u_{\varepsilon}+\left(\xi_{\varepsilon}-C_{\varepsilon}\right) u_{\varepsilon}\right]=\mathrm{e}^{-S_{\varepsilon}} \Lambda\left(\mathrm{e}^{S_{\varepsilon}} v_{\varepsilon}\right)+\left(\xi_{\varepsilon}-C_{\varepsilon}\right) v_{\varepsilon} .
$$

It is straightforward to verify that

$$
\mathrm{e}^{-S_{\varepsilon}} \Lambda\left(\mathrm{e}^{S_{\varepsilon}} v_{\varepsilon}\right)=\Lambda v_{\varepsilon}+v_{\varepsilon} \Lambda S_{\varepsilon}+\Xi_{\varepsilon} v_{\varepsilon}+\widetilde{Z}_{\varepsilon} v_{\varepsilon}
$$

Also, $\Lambda S_{\varepsilon}=-F * \xi_{\varepsilon}-\xi_{\varepsilon}$ by the definition of $F$. Thus we have

$$
\partial_{t} v_{\varepsilon}=\Lambda v_{\varepsilon}+v_{\varepsilon} \Lambda S_{\varepsilon}+\Xi_{\varepsilon} v_{\varepsilon}+\widetilde{Z}_{\varepsilon} v_{\varepsilon}+\left(\xi_{\varepsilon}-C_{\varepsilon}\right) v_{\varepsilon}=\Lambda v_{\varepsilon}+\left[-F * \xi_{\varepsilon}+Z_{\varepsilon}\right] v_{\varepsilon}+\Xi_{\varepsilon} v_{\varepsilon}
$$

The definitions of $S_{\varepsilon}$ and $\Xi_{\varepsilon}$ make sense for $\varepsilon=0$ as well. We let $S=S_{0}$ and $\Xi=\Xi_{0}$.

\footnotetext{
${ }^{1}$ We cannot quite apply [10, Lemma 5.5] as stated, since the Cauchy kernel cannot be extended by zero at negative times to a smooth function on $\mathbf{R}^{2} \backslash\{0\}$. However, this property is not necessary for our application.
} 


\section{Analytic estimates}

In this section we derive some purely analytic estimates that will help us control the quantities on the right side of (3.1). Following [11, Section 3] or [10, Section 10.3], define the norm, for any $m \in \mathbf{N}, \zeta \in \mathbf{R}$, and smooth function $K$ on $\mathbf{R} \backslash\{0\}$,

$$
\|K\|_{\zeta ; m}=\sup _{\substack{k \in \mathbf{Z} \\ 0 \leq k \leq m}} \sup _{x \in \mathbf{R} \backslash\{0\}}|x|^{k-\zeta}\left|K^{(k)}(x)\right|,
$$

where $K^{(k)}$ denotes the $k$ th derivative of $K$. We note in particular that, with $G$ defined as in Lemma 2.3, we have

$$
\|G\|_{-\kappa ; m}<\infty
$$

for all $\kappa>0$ and all $m \in \mathbf{N}$. We define the notation

$$
\square K(\alpha ; y, z)=K(\alpha)-K(\alpha-y)-K(\alpha-z)+K(\alpha-y-z) .
$$

Quantities of this form arise in the expressions for moments of (3.2)-(3.3).

Lemma 4.1. For each $\theta \in(0,1)$, there is a constant $C<\infty$ so that for any smooth function $K$ on $\mathbf{R} \backslash\{0\}$ and $\alpha, y, z \in \mathbf{R}$, we have

$$
|\square K(\alpha ; y, z)| \leq C\|K\|_{1-\theta ; 1}(|y| \wedge|z|)^{1-\theta}
$$

If we further assume that $|y|,|z|<|\alpha| / 4$, then

$$
|\square K(\alpha ; y, z)| \leq C|y||z||| K \|_{1-\theta ; 2}|\alpha|^{-1-\theta} .
$$

Proof. By the fundamental theorem of calculus and (4.1), we have for $x<w$ that

$$
|K(w)-K(x)| \leq \int_{x}^{w}\|K\|_{1-\theta ; 1}|t|^{-\theta} \mathrm{d} t \leq\|K\|_{1-\theta ; 1} \int_{-\frac{w-x}{2}}^{\frac{w-x}{2}}|t|^{-\theta} \mathrm{d} t \leq C\|K\|_{1-\theta ; 1}|w-x|^{1-\theta} .
$$

Thus by the triangle inequality we have

$$
|\square K(\alpha ; y, z)| \leq|K(\alpha)-K(\alpha-y)|+|K(\alpha-z)-K(\alpha-y-z)| \leq C\|K\|_{1-\theta ; 1}|y|^{1-\theta},
$$

and similarly with $y$ and $z$ exchanged. This proves (4.4). Now assume that $|y|,|z|<|\alpha| / 4$. If $F(w, x)=K(\alpha-w-x)$, then

$$
\begin{aligned}
|\square K(\alpha ; y, z)| & =\left|\int_{0}^{y} \int_{0}^{z} K^{\prime \prime}(\alpha-w-x) \mathrm{d} w \mathrm{~d} x\right| \\
& \leq\|K\|_{1-\theta ; 2}\left|\int_{0}^{y} \int_{0}^{z}(\alpha-w-x)^{-1-\theta} \mathrm{d} w \mathrm{~d} x\right| \leq C|y||z|\|K\|_{1-\theta ; 2}|\alpha|^{-1-\theta} .
\end{aligned}
$$

This proves (4.5).

Lemma 4.2. For each $\theta \in(0,1)$ and all $M<\infty$, there is a constant $C<\infty$ so that for any smooth functions $H_{1}, H_{2}: \mathbf{R} \backslash\{0\} \rightarrow \mathbf{R}$ and all $\alpha \in \mathbf{R}$ we have

$$
\int_{\mathbf{R}^{2}} \frac{\square H_{1}(\alpha ; y, z) \cdot \square H_{2}(\alpha ; y, z)}{y^{2} z^{2}} \mathrm{~d} y \mathrm{~d} z \leq C\left\|H_{1}\right\|_{1-\theta ; 2}\left\|H_{2}\right\|_{1-\theta ; 2}|\alpha|^{-2 \theta} .
$$

Proof. The left side of (4.6) can be written as $I_{1}+I_{2}$, where $I_{1}$ is the integral over the domain $\{|y|,|z|<|\alpha| / 4\}$ and $I_{2}$ is the integral over the domain $\{|y| \vee|z| \geq \alpha / 4\}$. By (4.5),

$$
I_{1} \leq C\left\|H_{1}\right\|_{1-\theta ; 2}\left\|H_{2}\right\|_{1-\theta ; 2}|\alpha|^{-2 \theta}
$$

while by (4.4),

$$
\frac{I_{2}}{\left\|H_{1}\right\|_{1-\theta ; 1}\left\|H_{2}\right\|_{1-\theta ; 1}} \leq C \int_{|\alpha| / 4 \leq|y| \vee|z|} \frac{(|y| \wedge|z|)^{2(1-\theta)}}{y^{2} z^{2}} \mathrm{~d} y \mathrm{~d} z \leq C|\alpha|^{-2 \theta} .
$$

Combining (4.7) and (4.8) yields (4.6). 


\section{Stability of the coefficients of the equation for $v_{\varepsilon}$}

In this section we prove that the coefficients of the equation (3.1) are stable as we eliminate the spatial mollification of the noise. We will consider the coefficients of (3.1) in turn. The stability of $F * \xi_{\varepsilon}$ will come directly from the decay (2.4) of $F$ and $F^{\prime}$. We will consider the term $Z_{\varepsilon}$, which requires renormalization, in Section 5.1. We bound the size of the renormalization constant $C_{\varepsilon}$ in Section 5.2. Then we show the stability of the nonlocal operator $\Xi_{\varepsilon}$ in Section 5.3. We will use the following two basic lemmas.

Lemma 5.1. For any $\kappa>0$ and $\varepsilon \geq 0$, we have that $\xi_{\varepsilon} \in \mathcal{C}^{-\frac{1}{2}-\kappa}$ almost surely. Also, $\xi_{\varepsilon} \rightarrow \xi$ as $\varepsilon \rightarrow 0$ in probability in $\mathcal{C}^{-\frac{1}{2}-\kappa}$.

Proof. This is a simple estimate using Proposition 2.1 as in [11, Lemma 1.1].

Lemma 5.2. For any $\kappa>0$, we have for each $\varepsilon \geq 0$ that $S_{\varepsilon} \in \mathcal{C}^{\frac{1}{2}-\kappa}$ almost surely. (Recall that $S_{\varepsilon}$ was defined in Lemma 3.1.) Also, $S_{\varepsilon} \rightarrow S$ as $\varepsilon \rightarrow 0$ in probability in $\mathcal{C}^{\frac{1}{2}-\kappa}$.

Proof. This follows from Lemma 5.1 by Lemma 2.4, similarly to [11, Corollary 1.2].

\subsection{Stability of $Z_{\varepsilon}$}

For $\varepsilon \geq 0$, define $S_{\varepsilon}(y, x)=S_{\varepsilon}(y)-S_{\varepsilon}(x)$. (Norms of the form $\left\|S_{\varepsilon}\right\|$ • will continue to refer to the one-variable function $S_{\varepsilon}$.) For $\varepsilon>0$, we fix

$$
C_{\varepsilon}=\frac{1}{2 \pi} \int \frac{\mathbf{E} S_{\varepsilon}(y, 0)^{2}}{y^{2}} \mathrm{~d} y
$$

We will prove in Proposition 5.9 below that the integrals on the right side of (5.1) are well-defined. For $\eta \in \mathcal{C}_{\mathrm{c}}^{1}$ and $\varepsilon \geq 0$, put

$$
Z_{\varepsilon}(\eta)=\frac{1}{\pi} \iint \frac{\eta(x)}{(y-x)^{2}}\left[\mathrm{e}^{S_{\varepsilon}(y, x)}-\left(1+S_{\varepsilon}(y, x)+\frac{1}{2} \mathbf{E} S_{\varepsilon}(y, x)^{2}\right)\right] \mathrm{d} y \mathrm{~d} x,
$$

so $Z_{\varepsilon}$ is a distribution. We will show that $Z_{\varepsilon} \in \mathcal{C}^{-\kappa}$ for any $\kappa>0$ and $\varepsilon \geq 0$ in Proposition 5.3 below. For $\varepsilon>0$, (5.2) agrees with (3.2) with the choice (5.1) of $C_{\varepsilon}$.

We split $Z_{\varepsilon}$ into two parts. For $\varepsilon \geq 0$ and $\eta \in \mathcal{C}_{\mathrm{c}}^{1}$, define

$$
U_{\varepsilon}(\eta)=\frac{1}{2 \pi} \iint \frac{\eta(x)}{(y-x)^{2}}\left[S_{\varepsilon}(y, x)^{2}-\mathbf{E} S_{\varepsilon}(y, x)^{2}\right] \mathrm{d} y \mathrm{~d} x,
$$

and for $\varepsilon \geq 0$ and $x \in \mathbf{R}$ define

$$
V_{\varepsilon}(x)=\frac{1}{\pi} \int \frac{\mathrm{e}^{S_{\varepsilon}(y, x)}-\left[1+S_{\varepsilon}(y, x)+\frac{1}{2} S_{\varepsilon}(y, x)^{2}\right]}{(y-x)^{2}} \mathrm{~d} y .
$$

Now evidently $Z_{\varepsilon}=U_{\varepsilon}+V_{\varepsilon}$. The main goal of this section is to prove the following.

Proposition 5.3. Let $\kappa>0$. For every $\varepsilon \geq 0$, we have $Z_{\varepsilon} \in \mathcal{C}^{-\kappa}$ almost surely. Moreover, $Z_{\varepsilon} \rightarrow Z_{0}$ in probability in $\mathcal{C}^{-\kappa}$.

To prove Proposition 5.3, we will show that $U_{\varepsilon}$ and $V_{\varepsilon}$ are both stable in $\mathcal{C}^{-\kappa}$ as $\varepsilon \rightarrow 0$. This is the advantage of the change of variables carried out in Section 3 , since the coefficients of the original equation (1.2) do not converge as $\varepsilon \rightarrow 0$. 


\subsubsection{Stability of $V_{\varepsilon}$}

First we consider $V_{\varepsilon}$, which is actually stable in $L^{\infty}$ as $\varepsilon \rightarrow 0$.

Lemma 5.4. For each $\varepsilon \geq 0$, we have $V_{\varepsilon} \in L^{\infty}$ with probability 1 . Also, $V_{\varepsilon} \rightarrow V_{0}$ in probability in $L^{\infty}$.

Proof. Let $f(x)=\mathrm{e}^{x}-\left(1+x+x^{2} / 2\right)$. By Taylor's theorem, we have a $C<\infty$ so that

$$
|f(x)| \leq C\left(|x|^{3} \wedge 1\right) \mathrm{e}^{|x|} ; \quad\left|f^{\prime}(x)\right| \leq C\left(|x|^{2} \wedge 1\right) \mathrm{e}^{|x|} .
$$

Thus, for all $\varepsilon \geq 0$ and all $x, y \in \mathbf{R}$,

$$
\left|\mathrm{e}^{S_{\varepsilon}(y, x)}-\left(1+S_{\varepsilon}(y, x)+\frac{1}{2} S_{\varepsilon}(y, x)^{2}\right)\right| \leq C \exp \left\{2\left\|S_{\varepsilon}\right\|_{\mathcal{C}^{\frac{1}{2}-\kappa}}\right\}\left(|y-x|^{\frac{3}{2}-3 \kappa} \wedge 1\right) .
$$

But this means that

$$
\left|V_{\varepsilon}(x)\right| \leq C \exp \left\{2\left\|S_{\varepsilon}\right\|_{\mathcal{C}^{\frac{1}{2}-\kappa}}\right\} \int \frac{|y-x|^{\frac{3}{2}-3 \kappa} \wedge 1}{(y-x)^{2}} \mathrm{~d} y,
$$

and the last integral is finite and independent of $x$ if $\kappa<\frac{1}{2}$. This implies that $V_{\varepsilon} \in L^{\infty}$.

Also, by the mean value theorem and (5.5), we have for all $x, y \in \mathbf{R}$ that

$$
\begin{aligned}
& \left|\mathrm{e}^{S_{\varepsilon}(y, x)}-\left(1+S_{\varepsilon}(y, x)+\frac{1}{2} S_{\varepsilon}(y, x)^{2}\right)-\left[\mathrm{e}^{S(y, x)}-\left(1+S(y, x)+\frac{1}{2} S(y, x)^{2}\right)\right]\right| \\
& \quad \leq C\left|S_{\varepsilon}(y, x)-S(y, x)\right|\left(\left(\left|S_{\varepsilon}(y, x)\right| \vee|S(y, x)|\right)^{2} \wedge 1\right) \exp \left\{\left|S_{\varepsilon}(y, x)\right| \vee|S(y, x)|\right\} \\
& \quad \leq C\left\|S_{\varepsilon}-S\right\|_{\mathcal{C}^{\frac{1}{2}-\kappa}}\left(|y-x|^{\frac{3}{2}-3 \kappa} \wedge 1\right) \exp \left\{2 \left(\left\|S_{\varepsilon}\right\|_{\mathcal{C}^{\frac{1}{2}-\kappa}} \vee\|S\|_{\left.\left.\mathcal{C}^{\frac{1}{2}-\kappa}\right)\right\} .}\right.\right.
\end{aligned}
$$

Therefore, for all $x \in \mathbf{R}$, we have

$$
\left|V_{\varepsilon}(x)-V(x)\right| \leq C\left\|S_{\varepsilon}-S\right\|_{\mathcal{C}^{\frac{1}{2}-\kappa}} \exp \left\{2\left(\left\|S_{\varepsilon}\right\|_{\mathcal{C}^{\frac{1}{2}-\kappa}} \vee\|S\|_{\mathcal{C}^{\frac{1}{2}-\kappa}}\right)\right\} \int \frac{|y-x|^{\frac{3}{2}-3 \kappa} \wedge 1}{(y-x)^{2}} \mathrm{~d} y .
$$

The integral is bounded independently of $x$, and by Lemma 5.2 we have

$$
\left\|S_{\varepsilon}-S\right\|_{\mathcal{C}^{\frac{1}{2}-\kappa}} \exp \left\{2\left(\left\|S_{\varepsilon}\right\|_{\mathcal{C}^{\frac{1}{2}-\kappa}} \vee\|S\|_{\mathcal{C}^{\frac{1}{2}-\kappa}}\right)\right\} \rightarrow 0
$$

in probability as $\varepsilon \rightarrow 0$. This proves that $V_{\varepsilon} \rightarrow V$ in probability in $L^{\infty}$ as $\varepsilon \rightarrow 0$.

\subsubsection{Stability of $U_{\varepsilon}$}

Now we show the stability of $U_{\varepsilon}$. Since $U_{\varepsilon}(\eta)$ is defined as an integral over squares of Gaussian random variables-elements of the second Wiener chaos-we can use moment estimates to control its regularity and establish its stability.

Lemma 5.5. For each $\kappa>0$, we have a constant $C$ so that for all $\varepsilon \in[0,1]$ and $\eta \in \mathcal{C}^{1}([-1,1])$ with $\|\eta\|_{\mathcal{C}^{0}} \leq 1$ we have, defining $\eta_{x}^{\lambda}$ as in (2.1), that $\mathbf{E} U_{\varepsilon}\left(\eta_{x}^{\lambda}\right)^{2} \leq C \lambda^{-\kappa}$.

Proof. Taking the second moment of (5.3), we have

$$
\mathbf{E} U_{\varepsilon}(\eta)^{2}=\frac{1}{\pi^{2}} \iiint \int \frac{\eta(x) \eta(w)}{(x-y)^{2}(w-z)^{2}} \operatorname{Cov}\left(S_{\varepsilon}(y, x)^{2}, S_{\varepsilon}(z, w)^{2}\right) \mathrm{d} y \mathrm{~d} z \mathrm{~d} x \mathrm{~d} w
$$

We can compute

$$
\begin{aligned}
\mathbf{E} S_{\varepsilon}(x, y) S_{\varepsilon}(w, z) & =\sum_{k \in \mathbf{Z}} \int\left(G_{\varepsilon}(x-r+k L)-G_{\varepsilon}(y-r+k L)\right)\left(G_{\varepsilon}(w-r)-G_{\varepsilon}(z-r)\right) \mathrm{d} r \\
& =\square H_{\varepsilon}(x-w ; x-y, z-w)
\end{aligned}
$$


where $H_{\varepsilon}(q)=\sum_{k \in \mathbf{Z}} G_{\varepsilon}^{* 2}(q+k L)$ and $\square$ is defined as in (4.3). By the Isserlis theorem,

$$
\operatorname{Cov}\left(S_{\varepsilon}(y, x)^{2}, S_{\varepsilon}(z, w)^{2}\right)=2\left(\mathbf{E} S_{\varepsilon}(y, x) S_{\varepsilon}(z, w)\right)^{2}=2\left(\square H_{\varepsilon}(x-w ; x-y, z-w)\right)^{2} .
$$

Using (4.2) and [10, (10.12)] and recalling that $G$ has compact support, we see that $\left\|H_{\varepsilon}-H_{\varepsilon}(0)\right\|_{1-\frac{\kappa}{2} ; m} \leq C$, where $C<\infty$ is a constant independent of $\varepsilon$. But the $\square$ operator does not see constants, so applying Lemma 4.2, we have

$$
\begin{aligned}
\mathbf{E} U_{\varepsilon}(\eta)^{2} & \leq \frac{2}{\pi^{2}} \iiint \int \eta(x) \eta(w)\left(\frac{\square H_{\varepsilon}(x-w ; x-y, z-w)}{(x-y)(w-z)}\right)^{2} \mathrm{~d} y \mathrm{~d} z \mathrm{~d} x \mathrm{~d} w \\
& \leq C \iint \eta(x) \eta(w)|x-w|^{-\kappa} \mathrm{d} x \mathrm{~d} w .
\end{aligned}
$$

Then the conclusion follows by rescaling.

Corollary 5.6. For any $\kappa>0$ and $\varepsilon \geq 0$, we have $U_{\varepsilon} \in \mathcal{C}^{-\kappa}$ almost surely.

Proof. We note that $U_{\varepsilon}$ is an element of the second Wiener chaos by definition. By Lemma 5.5 and the equivalence of moments of elements of finite Wiener chaoses (as stated for example in [10, Lemma 10.5]) and Lemma 5.5, for each $\kappa>0$ and $p \in[1, \infty)$ there is a constant $C=C(p, \kappa)<\infty$, depending only on $p$ and $\kappa$, so that

$$
\mathbf{E} \sup _{\substack{x \in \mathbf{Z} \\ n \in \mathbf{N}}} 2^{-\kappa n p}\left|U_{\varepsilon}\left(\psi_{2^{-n} x}^{2^{-n}}\right)\right|^{p} \leq \sum_{n \in \mathbf{N}} \sum_{x=0}^{2^{n} L} 2^{-\kappa n p} \mathbf{E}\left|U_{\varepsilon}\left(\psi_{2^{-n} x}^{2^{-n}}\right)\right|^{p} \leq C L \sum_{n \in \mathbf{N}} 2^{n[1+\kappa-\kappa p / 2]} .
$$

Choose $p>2(1 / \kappa+1)$, so the last sum is finite. It is simpler to show that $\mathbf{E} \sup _{x \in \mathbf{Z}}\left|U_{\varepsilon}\left(\phi_{x}^{1}\right)\right|^{p}<$ $\infty$. By Proposition 2.1, this means that $\mathbf{E}\left\|U_{\varepsilon}\right\|_{\mathcal{C}^{-\kappa}}^{p}<\infty$, so $\left\|U_{\varepsilon}\right\|_{\mathcal{C}^{-\kappa}}<\infty$ almost surely.

Lemma 5.7. For any $\kappa \in(0,1)$ and $R>0$, we have a constant $C<\infty$ so that, for any $\eta \in \mathcal{C}^{1}([-R, R])$ with $\|\eta\|_{\mathcal{C}^{0}} \leq 1$ and any $x_{0} \in \mathbf{R}$, we have

$$
\mathbf{E}\left[U_{\varepsilon}\left(\eta_{x_{0}}^{\lambda}\right)-U\left(\eta_{x_{0}}^{\lambda}\right)\right]^{2} \leq C \varepsilon^{\kappa / 6} \lambda^{-\kappa} .
$$

Proof. For any $\eta \in \mathcal{C}_{\mathrm{c}}^{1}$, we have

$$
U_{\varepsilon}(\eta)-U(\eta)=\frac{1}{2 \pi} \iint \eta(x) \frac{S_{\varepsilon}(y, x)^{2}-S(y, x)^{2}-\mathbf{E}\left(S_{\varepsilon}(y, x)^{2}-S(y, x)^{2}\right)}{(y-x)^{2}} \mathrm{~d} y \mathrm{~d} x,
$$

so

$$
\begin{array}{r}
\mathbf{E}\left(U_{\varepsilon}(\eta)-U(\eta)\right)^{2}=\frac{1}{4 \pi^{2}} \iiint \int \frac{\operatorname{Cov}\left(S_{\varepsilon}(y, x)^{2}-S(y, x)^{2}, S_{\varepsilon}(z, w)^{2}-S(z, w)^{2}\right)}{(x-y)^{2}(w-z)^{2}} \\
\eta(x) \eta(w) \mathrm{d} y \mathrm{~d} z \mathrm{~d} x \mathrm{~d} w .
\end{array}
$$

By the Isserlis theorem we have that

$$
\begin{aligned}
& \operatorname{Cov}\left(S_{\varepsilon}(y, x)^{2}-S(y, x)^{2}, S_{\varepsilon}(z, w)^{2}-S(z, w)^{2}\right) \\
&= \mathbf{E}\left[\left(S_{\varepsilon}+S\right)(y, x)\left(S_{\varepsilon}-S\right)(z, w)\right] \mathbf{E}\left[\left(S_{\varepsilon}-S\right)(y, x)\left(S_{\varepsilon}+S\right)(z, w)\right] \\
&+\mathbf{E}\left[\left(S_{\varepsilon}+S\right)(y, x)\left(S_{\varepsilon}+S\right)(z, w)\right] \mathbf{E}\left[\left(S_{\varepsilon}-S\right)(y, x)\left(S_{\varepsilon}-S\right)(z, w)\right] \\
&=\left(\left(\square\left[\mathcal{P}\left[\left(G_{\varepsilon}+G\right) *\left(G_{\varepsilon}-G\right)\right]\right]\right)^{2}+\square\left[\mathcal{P}\left[\left(G_{\varepsilon}+G\right)^{* 2}\right] \square\left[\mathcal{P}\left[\left(G_{\varepsilon}-G\right)^{* 2}\right]\right]\right)\right. \\
&(x-w ; x-y, z-w),
\end{aligned}
$$


where $\mathcal{P} f(x)=\sum_{k \in \mathbf{Z}} f(x+k L)$. Combining this with (5.9) yields

$$
\begin{gathered}
\mathbf{E}\left[U(\eta)-U_{\varepsilon}(\eta)\right]^{2} \leq C \iiint \int\left[\left(\square\left[\mathcal{P}\left[\left(G_{\varepsilon}+G\right) *\left(G_{\varepsilon}-G\right)\right](x-w ;-y, z)\right)^{2}\right.\right. \\
\left.+\left(\square\left[\mathcal{P}\left[\left(G_{\varepsilon}+G\right)^{* 2}\right]\right] \cdot \square\left[\mathcal{P}\left[\left(G_{\varepsilon}-G\right)^{* 2}\right]\right]\right)(x-w ;-y, z)\right] \\
y^{-2} z^{-2} \eta(x) \eta(w) \mathrm{d} y \mathrm{~d} z \mathrm{~d} x \mathrm{~d} w
\end{gathered}
$$

By [10, Lemma 10.17] and (4.2), for any $m \in \mathbf{Z}_{\geq 0}$ we have a constant $C$, independent of $\varepsilon$, so that $\left\|G_{\varepsilon}\right\|_{-2 \kappa ; m} \leq C$ and $\left\|G_{\varepsilon}-G\right\|_{-2 \kappa ; m} \leq C \varepsilon^{\kappa}$. By [10, (10.12)], we thus obtain

$$
\begin{aligned}
\left\|\mathcal{P}\left[\left(G_{\varepsilon}-G\right) *\left(G_{\varepsilon}+G\right)\right]-\mathcal{P}\left[\left(G_{\varepsilon}-G\right) *\left(G_{\varepsilon}+G\right)\right](0)\right\|_{1-3 \kappa ; m} & \leq C \varepsilon^{\kappa} \\
\left\|\mathcal{P}\left[\left(G_{\varepsilon}-G\right)^{* 2}\right]-\left(G_{\varepsilon}-G\right)^{* 2}(0)\right\|_{1-3 \kappa ; m} & \leq C \varepsilon^{2 \kappa} \\
\left\|\mathcal{P}\left[\left(G_{\varepsilon}+G\right)^{* 2}\right]-\left(G_{\varepsilon}+G\right)^{* 2}(0)\right\|_{1-3 \kappa ; m} & \leq C .
\end{aligned}
$$

The operator $\square$ does not see constants, so by these inequalities, (5.10), and Lemma 4.2,

$$
\mathbf{E}\left[U(\eta)-U_{\varepsilon}(\eta)\right]^{2} \leq C \varepsilon^{\kappa} \int \eta(x) \eta(w)|x-w|^{-6 \kappa} \mathrm{d} x \mathrm{~d} w .
$$

Then (5.8) follows by substituting $\kappa \mapsto \kappa / 6$ and rescaling. The assumption $\kappa<1$ is so that the integral on the right side of (5.11) can be bounded in terms of $\|\eta\|_{\mathcal{C}^{0}}$ and $R$.

Corollary 5.8. For any $\kappa>0, U_{\varepsilon} \rightarrow U$ in probability in $\mathcal{C}^{-\kappa}$.

Proof. Assume without loss of generality that $\kappa<1$. As in Corollary 5.6, $U_{\varepsilon}-U$ is an element of the second Wiener chaos by definition. By Lemma 5.7 and the equivalence of moments of the elements of finite Wiener chaoses, for each $\kappa>0$ and $p \in[1, \infty)$ there is a constant $C=C(p, \kappa)$, depending only on $p$ and $\kappa$, so that

$$
\mathbf{E} \sup _{\substack{x \in \mathbf{Z} \\ n \in \mathbf{N}}} \frac{\left|\left(U_{\varepsilon}-U\right)\left(\psi_{2^{n} x}^{2^{-n}}\right)\right|^{p}}{2^{\kappa n p}} \leq \sum_{n \in \mathbf{N}} \sum_{x=0}^{2^{n} L} \frac{\mathbf{E}\left|\left(U_{\varepsilon}-U\right)\left(\psi_{2^{-n} x}^{2^{-n}}\right)\right|^{p}}{2^{\kappa n p}} \leq C L \varepsilon^{\frac{\kappa p}{6}} \sum_{n \in \mathbf{N}} 2^{n\left[1+\kappa-\frac{\kappa p}{2}\right]} .
$$

Take $p>2(1 / \kappa+1)$, so the last sum is finite. A simpler computation shows that $\mathbf{E} \sup _{x \in \mathbf{Z}}\left|\left(U_{\varepsilon}-U\right)\left(\phi_{x}^{1}\right)\right|^{p} \leq C \varepsilon^{\kappa p / 6}$. By Proposition 2.1, E $\left\|U_{\varepsilon}-U\right\|_{\mathcal{C}^{-\kappa}}^{p} \leq C \varepsilon^{\kappa p / 6}$ for some constant $C$ not depending on $\varepsilon$, which means that $U_{\varepsilon} \rightarrow U$ in probability.

The results of the last two subsections are now enough to prove Proposition 5.3.

Proof of Proposition 5.3. Since $Z_{\varepsilon}=U_{\varepsilon}+V_{\varepsilon}$ for all $\varepsilon \geq 0$, the fact that $Z_{\varepsilon} \in \mathcal{C}^{-\kappa}$ almost surely is an immediate consequence of Lemma 5.4 and Corollary 5.6, and the convergence is an immediate consequence of Lemmas 5.4 and 5.7.

\subsection{The renormalization constant}

We now estimate the size of the renormalization constant $C_{\varepsilon}$, proving the bound (1.4).

Proposition 5.9. There is an absolute constant $C$ so that, for all $\varepsilon \in(0,1], \mid C_{\varepsilon}-$ $(1 / \pi) \log (1 / \varepsilon) \mid \leq C$.

Proof. By (5.7), we have (with $H_{\varepsilon}$ as defined there)

$$
\mathbf{E} S_{\varepsilon}(y, 0)^{2}=\square H_{\varepsilon}(0 ;-y, y)=2\left(H_{\varepsilon}(0)-H_{\varepsilon}(y)\right) .
$$

We note that $H_{\varepsilon}$ is even so $H_{\varepsilon}^{\prime}(0)=0$. Thus, combining (2.2), (5.1), and (5.12) we obtain

$$
C_{\varepsilon}=-\Lambda H_{\varepsilon}(0)=C-\Lambda\left(G^{* 2} * \rho_{\varepsilon}^{* 2}\right)(0)=C-\frac{1}{\pi} \log |\cdot| * \rho_{\varepsilon}^{* 2}(0)-F * G * \rho_{\varepsilon}^{* 2}(0) .
$$


Here, $C$ is independent of $\varepsilon$, coming from the terms $k \neq 0$ in the sum defining $H_{\varepsilon}$. The third term in (5.13) is also bounded independently of $\varepsilon$, while the second is

$$
-\frac{1}{\pi} \log |\cdot| * \rho_{\varepsilon}^{* 2}(0)=-\frac{1}{\pi} \int \log |x| \rho^{* 2}(x / \varepsilon) \mathrm{d} x=-\frac{1}{\pi} \int \log |\varepsilon x| \rho^{* 2}(-x) \mathrm{d} x=\frac{1}{\pi} \log \frac{1}{\varepsilon}+C .
$$

\subsection{Stability of $\Xi_{\varepsilon}$}

In this section, we show that $\Xi_{\varepsilon}$ (defined in (3.3)) is stable as $\varepsilon \rightarrow 0$.

Lemma 5.10. If $\kappa>0$ and $\varepsilon \geq 0$, the map $\Xi_{\varepsilon}$ is almost surely a bounded linear map $\mathcal{C}^{\frac{1}{2}+\kappa} \rightarrow L^{\infty}$.

Proof. We have

$$
\sup _{x, y \in \mathbf{R}} \frac{\left|\mathrm{e}^{S_{\varepsilon}(y, x)}-1\right|}{|y-x|^{\frac{1}{2}-\frac{\kappa}{2}}} \leq 1+\exp \left\{2\left\|S_{\varepsilon}\right\|_{\mathcal{C}^{\frac{1}{2}-\frac{\kappa}{2}}}\right\}
$$

so by the triangle inequality,

$$
\left|\Xi_{\varepsilon} v(x)\right| \leq C\left(1+\exp \left\{2\left\|S_{\varepsilon}\right\|_{\mathcal{C}^{\frac{1}{2}-\frac{\kappa}{2}}}\right\}\right)\|v\|_{\mathcal{C}^{\frac{1}{2}+\kappa}} \int \frac{|x-y|^{\frac{1}{2}-\frac{\kappa}{2}}\left(|x-y|^{\frac{1}{2}+\kappa} \wedge 1\right)}{(y-x)^{2}} \mathrm{~d} y,
$$

and the integral is bounded independently of $x$.

Proposition 5.11. For any $\kappa>0$, we have $\Xi_{\varepsilon} \rightarrow \Xi$ in probability with respect to the norm topology of the space $\mathcal{B}\left(\mathcal{C}^{\frac{1}{2}+\kappa}, L^{\infty}\right)$ of bounded linear operators from $\mathcal{C}^{\frac{1}{2}+\kappa}$ to $L^{\infty}$.

Proof. We have for all $x, y \in \mathbf{R}$ that

$$
\left|\mathrm{e}^{S_{\varepsilon}(y, x)}-\mathrm{e}^{S(y, x)}\right| \leq 2 \exp \left\{2\left\|S_{\varepsilon}\right\|_{L^{\infty}}+2\|S\|_{L^{\infty}}\right\}\left\|S_{\varepsilon}-S\right\|_{\mathcal{C}^{\frac{1}{2}-\frac{\kappa}{2}}}|y-x|^{\frac{1}{2}-\frac{\kappa}{2}},
$$

so by Lemma 5.2, we have $\sup _{x, y \in \mathbf{R}} \frac{\left|\mathrm{e}^{S_{\varepsilon}(y, x)}-\mathrm{e}^{S(y, x)}\right|}{|y-x|^{\frac{1}{2}-\frac{\kappa}{2}}} \rightarrow 0$ in probability as $\varepsilon \rightarrow 0$. Now we write, for any $v \in \mathcal{C}^{\frac{1}{2}+\kappa}$,

$$
\begin{aligned}
\left|\left(\Xi-\Xi_{\varepsilon}\right) v(x)\right| & \leq \frac{1}{\pi}\left|\int \frac{\left(e^{S_{\varepsilon}(y, x)}-e^{S(y, x)}\right)(v(y)-v(x))}{(y-x)^{2}} \mathrm{~d} y\right| \\
& \leq C\|v\|_{\mathcal{C}^{\frac{1}{2}+\kappa}}\left(\sup _{x, y \in \mathbf{R}} \frac{\left|e^{S_{\varepsilon}(y, x)}-e^{S(y, x)}\right|}{|y-x|^{\frac{1}{2}-\frac{\kappa}{2}}}\right) \int \frac{\left(|y-x|^{\frac{1}{2}+\kappa} \wedge 1\right)|y-x|^{\frac{1}{2}-\frac{\kappa}{2}}}{(y-x)^{2}} \mathrm{~d} y .
\end{aligned}
$$

The right side is finite and independent of $v$, and as $\varepsilon \rightarrow 0$ converges to 0 in probability.

\section{The fixed-point argument}

Fix $\kappa \in(0,1 / 4)$ and $T>0$, and define $\mathcal{X}_{T}^{\kappa}(\mathcal{Y})$ for any Banach space $\mathcal{Y}$ as in the introduction. We will construct a solution to (3.1) in the space $\mathcal{X}_{T}^{\kappa}\left(\mathcal{C}^{\frac{1}{2}+\kappa}\right)$ using a fixedpoint argument. For $g \in \mathcal{C}^{-\kappa}, \Psi \in \mathcal{B}\left(\mathcal{C}^{\frac{1}{2}+\kappa}, \mathcal{C}^{-\kappa}\right)$, and $\underline{v} \in \mathcal{C}^{-\frac{1}{2}+2 \kappa}$, define the affine operator $\mathcal{M}_{g, \Psi, \underline{v}}$ on $\mathcal{X}_{T}^{\kappa}\left(\mathcal{C}^{\frac{1}{2}+\kappa}\right)$ (see Corollary 6.2 below) by

$\mathcal{M}_{g, \Psi, \underline{v}} v(t, x)=\mathcal{L}_{g, \Psi} v(t, x)+P_{t} * \underline{v}(x)$, with $\mathcal{L}_{g, \Psi} v(t, x)=\int_{0}^{t} P_{t-s} *(v(s, \cdot) \cdot g+\Psi v(s, \cdot)) \mathrm{d} s$.

Recall that the fractional heat kernel $P_{t}$ was introduced before Lemma 2.5. Solutions $v_{\varepsilon}$ of (3.1) are exactly fixed points of the map $\mathcal{M}_{Z_{\varepsilon}-F * \xi_{\varepsilon}, \Xi_{\varepsilon}, \underline{v}_{\varepsilon}}$. We aim to show that there is a unique such fixed point. We start by bounding the operator norm of $\mathcal{L}_{g, \Psi}$.

Lemma 6.1. We have a $C<\infty$ so that if $g \in \mathcal{C}^{-\kappa}$ and $\Psi \in \mathcal{B}\left(\mathcal{C}^{\frac{1}{2}+\kappa}, \mathcal{C}^{-\kappa}\right)$, then $\mathcal{L}_{g, \Psi} \in$ $\mathcal{B}\left(\mathcal{X}_{T}^{\kappa}\left(\mathcal{C}^{\frac{1}{2}+\kappa}\right)\right)$ and $\left\|\mathcal{L}_{g, \Psi}\right\|_{\mathcal{B}\left(\mathcal{X}_{T}^{\kappa}\left(\mathcal{C}^{\frac{1}{2}+\kappa}\right)\right)} \leq C\left(\|g\|_{\mathcal{C}^{-\kappa}}+\|\Psi\|_{\mathcal{B}\left(\mathcal{C}^{\frac{1}{2}+\kappa}, \mathcal{C}^{-\kappa}\right)}\right) T^{\frac{1}{2}-2 \kappa}$. 
Proof. By Lemmas 2.5 and 2.2, we have a constant $C<\infty$ so that, for $v \in \mathcal{X}_{T}^{\kappa}\left(\mathcal{C}^{\frac{1}{2}+\kappa}\right)$,

$$
\begin{aligned}
& \left\|\mathcal{L}_{g, \Psi} v(t, \cdot)\right\|_{\mathcal{C}^{\frac{1}{2}+\kappa}} \leq C \int_{0}^{t}(t-s)^{-\frac{1}{2}-2 \kappa}\left(\|v(s, \cdot) \cdot g\|_{\mathcal{C}^{-\kappa}}+\|\Psi v(s, \cdot)\|_{\mathcal{C}^{-\kappa}}\right) \mathrm{d} s ; \\
& \|v(s, \cdot) \cdot g\|_{\mathcal{C}^{-\kappa}} \leq C s^{-1+\kappa}\|g\|_{\mathcal{C}^{-\kappa}}\|v\|_{\mathcal{X}_{T}^{\kappa}\left(\mathcal{C}^{\frac{1}{2}+\kappa}\right)} ; \\
& \|\Psi v(s, \cdot)\|_{\mathcal{C}^{-\kappa}} \leq s^{-1+\kappa}\|\Psi\|_{\mathcal{B}\left(\mathcal{C}^{\frac{1}{2}+\kappa}, \mathcal{C}^{-\kappa}\right)}\|v\|_{\mathcal{X}_{T}^{\kappa}\left(\mathcal{C}^{\frac{1}{2}+\kappa}\right)} .
\end{aligned}
$$

Plugging (6.2) and (6.3) into (6.1) and integrating (using that $\kappa<1 / 4$ ), we obtain

$$
\left\|\mathcal{L}_{g, \Psi} v(t, \cdot)\right\|_{\mathcal{C}^{\frac{1}{2}+\kappa}} \leq C\|v\|_{\mathcal{X}_{T}^{\kappa}\left(\mathcal{C}^{\frac{1}{2}+\kappa}\right)}\left(\|g\|_{\mathcal{C}^{-\kappa}}+\|\Psi\|_{\mathcal{B}\left(\mathcal{C}^{\frac{1}{2}+\kappa}, \mathcal{C}^{-\kappa}\right)}\right) t^{-\frac{1}{2}-\kappa}
$$

Then the conclusion follows from the definition (1.3) of the $\mathcal{X}_{T}^{\kappa}\left(\mathcal{C}^{\frac{1}{2}+\kappa}\right)$ norm.

If $T$ is chosen sufficiently small, Lemma 6.1 implies that $\mathcal{M}_{g, \Psi, \underline{v}}$ is a contraction map:

Corollary 6.2. There is a $C<\infty$ so that for any $g \in \mathcal{C}^{-\kappa}, \Psi \in \mathcal{B}\left(\mathcal{C}^{\frac{1}{2}+\kappa}, \mathcal{C}^{-\kappa}\right)$, and $\underline{v} \in \mathcal{C}^{-\frac{1}{2}+2 \kappa}$, the map $\mathcal{M}_{g, \Psi, \underline{v}}: \mathcal{X}_{T}^{\kappa}\left(\mathcal{C}^{\frac{1}{2}+\kappa}\right) \rightarrow \mathcal{X}_{T}^{\kappa}\left(\mathcal{C}^{\frac{1}{2}+\kappa}\right)$ is continuous, and if

$$
T<\left[C\left(\|g\|_{\mathcal{C}^{-\kappa}}+\|\Psi\|_{\mathcal{B}\left(\mathcal{C}^{\frac{1}{2}+\kappa}, \mathcal{C}^{-\kappa}\right)}\right)\right]^{-1 /(1 / 2-2 \kappa)}=: T_{0}\left(\|g\|_{\mathcal{C}^{-\kappa}},\|\Psi\|_{\mathcal{B}\left(\mathcal{C}^{\frac{1}{2}+\kappa}, \mathcal{C}^{-\kappa}\right)}\right),
$$

then $\mathcal{M}_{g, \Psi, \underline{v}}$ is a contraction map.

Proof. If $v \in \mathcal{X}_{T}^{\kappa}\left(\mathcal{C}^{\frac{1}{2}+\kappa}\right)$, then we have $\mathcal{L}_{g, \Psi} v \in \mathcal{X}_{T}^{\kappa}\left(\mathcal{C}^{\frac{1}{2}+\kappa}\right)$ by Lemma 6.1. By Lemma 2.5, we have a constant $C$ so that $\left\|P_{t} * \underline{v}\right\|_{\mathcal{C}^{\frac{1}{2}+\kappa}} \leq C t^{-1+\kappa}\|\underline{v}\|_{\mathcal{C}^{-\frac{1}{2}+2 \kappa}}$. This implies that $t \mapsto P_{t} * \underline{v}$ is an element of $\mathcal{X}_{T}^{\kappa}\left(\mathcal{C}^{\frac{1}{2}+\kappa}\right)$ as well. Therefore, $\mathcal{M}_{g, \Psi, \underline{v}} v \in \mathcal{X}_{T}^{\kappa}\left(\mathcal{C}^{\frac{1}{2}+\kappa}\right)$. Since $\mathcal{M}_{g, \Psi, \underline{v}} v-$ $\mathcal{M}_{g, \Psi, \underline{v}} \tilde{v}=\mathcal{L}_{g, \Psi} v-\mathcal{L}_{g, \Psi} \tilde{v}$, the continuity and contraction come from Lemma 6.1.

We now use the contraction mapping principle to construct fixed points of $\mathcal{M}_{g, \Psi, \underline{v}}$.

Lemma 6.3. For any $T<\infty, \mathcal{M}_{g, \Psi, \underline{v}}$ has a unique fixed point $\mathcal{V}_{T}(g, \Psi, \underline{v})$ in $\mathcal{X}_{T}^{\kappa}\left(\mathcal{C}^{\frac{1}{2}+\kappa}\right)$.

Proof. This holds for $T<T_{0}(g, \Psi)$ by Corollary 6.2. As $T_{0}(g, \Psi)$ does not depend on $\underline{v}$, the construction can be extended to all $T$ as in the proof of [11, Proposition 4.1].

Now, as in [11, Proposition 4.2], we show that the solution map $\mathcal{V}_{T}$ is continuous using a mild solution argument.

Proposition 6.4. For $T<\infty$, the map $\mathcal{V}_{T}: \mathcal{C}^{-\kappa} \times \mathcal{B}\left(\mathcal{C}^{\frac{1}{2}+\kappa}, \mathcal{C}^{-\kappa}\right) \times \mathcal{C}^{-\frac{1}{2}+2 \kappa} \rightarrow \mathcal{X}_{T}^{\kappa}\left(\mathcal{C}^{\frac{1}{2}+\kappa}\right)$ is continuous.

Proof. Let $M>0$ be arbitrary. We will show that $\mathcal{V}_{T}$ is continuous on $A_{M}=\{(g, \Psi, \underline{v})$ : $\left.\|g\|_{\mathcal{C}^{-\kappa}},\|\Psi\|_{\mathcal{B}\left(\mathcal{C}^{\frac{1}{2}+\kappa}, \mathcal{C}^{-\kappa}\right)} \leq M\right\}$. First suppose that $T<T_{0}(M, M)$. For $(g, \Psi, \underline{v}),(\tilde{g}, \tilde{\Psi}, \underline{\tilde{v}}) \in$ $A_{M}$, put $v=\mathcal{V}_{T}(g, \Psi, \underline{v})$ and $\tilde{v}=\mathcal{V}_{T}(\tilde{g}, \tilde{\Psi}, \underline{\tilde{v}})$, so

$(v-\tilde{v})(t, \cdot)=\mathcal{L}_{g, \Psi}(v-\tilde{v})(t, \cdot)+\int_{0}^{t} P_{t-s} *[\tilde{v}(s, \cdot)(g-\tilde{g})+(\Psi-\tilde{\Psi}) \tilde{v}(s, \cdot)] \mathrm{d} s+P_{t} *(\underline{v}-\underline{\tilde{v}})$.

Thus for all $t \in(0, T]$ we have

$$
\begin{aligned}
\|(v-\tilde{v})(t, \cdot)\|_{\mathcal{C}^{\frac{1}{2}+\kappa}} \leq\left\|\mathcal{L}_{g, \Psi}(v-\tilde{v})(t, \cdot)\right\|_{\mathcal{C}^{\frac{1}{2}+\kappa}}+C t^{-1+\kappa}\|\underline{v}-\underline{\tilde{v}}\|_{\mathcal{C}^{-\frac{1}{2}+2 \kappa}} \\
\quad+C\left(\|g-\tilde{g}\|_{\mathcal{C}^{-\kappa}}+\|\Psi-\tilde{\Psi}\|_{\mathcal{B}\left(\mathcal{C}^{\frac{1}{2}+\kappa}, \mathcal{C}^{-\kappa}\right)}\right) \int_{0}^{t}(t-s)^{-\frac{1}{2}-2 \kappa}\|\tilde{v}(s, \cdot)\|_{\mathcal{C}^{\frac{1}{2}+\kappa}} \mathrm{d} s .
\end{aligned}
$$


Therefore,

$$
\begin{aligned}
\|v-\tilde{v}\|_{\mathcal{X}_{T}^{\kappa}\left(\mathcal{C}^{\frac{1}{2}+\kappa}\right)} \leq & \left\|\mathcal{L}_{g, \Psi}\right\|_{\mathcal{B}\left(\mathcal{X}_{T}^{\kappa}\left(\mathcal{C}^{\frac{1}{2}+\kappa}\right)\right)}\|v-\tilde{v}\|_{\mathcal{X}_{T}^{\kappa}\left(\mathcal{C}^{\frac{1}{2}+\kappa}\right)}+C\|\underline{v}-\underline{\tilde{v}}\|_{\mathcal{C}^{-\frac{1}{2}+2 \kappa}} \\
& +C\left(\|g-\tilde{g}\|_{\mathcal{C}^{-\kappa}}+\|\Psi-\tilde{\Psi}\|_{\mathcal{B}\left(\mathcal{C}^{\frac{1}{2}+\kappa}, \mathcal{C}^{-\kappa}\right)}\right)\|\tilde{v}\|_{\mathcal{X}_{T}^{\kappa}\left(\mathcal{C}^{\frac{1}{2}+\kappa}\right)} \int_{0}^{t} \frac{s^{-1+\kappa}}{(t-s)^{\frac{1}{2}+2 \kappa}} \mathrm{d} s \\
\leq & \left\|\mathcal{L}_{g, \Psi}\right\|_{\mathcal{B}\left(\mathcal{X}_{T}^{\kappa}\left(\mathcal{C}^{\frac{1}{2}+\kappa}\right)\right)}\|v-\tilde{v}\|_{\mathcal{X}_{T}^{\kappa}\left(\mathcal{C}^{\frac{1}{2}+\kappa}\right)}+C\|\underline{v}-\underline{\tilde{v}}\|_{\mathcal{C}^{-\frac{1}{2}+2 \kappa}} \\
& +C M\left(\|g-\tilde{g}\|_{\mathcal{C}^{-\kappa}}+\|\Psi-\tilde{\Psi}\|_{\mathcal{B}\left(\mathcal{C}^{\frac{1}{2}+\kappa}, \mathcal{C}^{-\kappa}\right)}\right) T^{\frac{1}{2}-\kappa} .
\end{aligned}
$$

Since $T<T_{0}(M, M)$, we have $\left\|\mathcal{L}_{g, \Psi}\right\|_{\mathcal{B}\left(\mathcal{X}_{T}^{\kappa}\left(\mathcal{C}^{\frac{1}{2}+\kappa}\right)\right)}<1$, so

$$
\|v-\tilde{v}\|_{\mathcal{X}_{T}^{\kappa}\left(\mathcal{C}^{\frac{1}{2}+\kappa}\right)} \leq C \cdot \frac{\|\underline{v}-\underline{\tilde{v}}\|_{\mathcal{C}^{-\frac{1}{2}+2 \kappa}}+M\left(\|g-\tilde{g}\|_{\mathcal{C}^{-\kappa}}+\|\Psi-\tilde{\Psi}\|_{\mathcal{B}\left(\mathcal{C}^{\frac{1}{2}+\kappa}, \mathcal{C}^{-\kappa}\right)}\right) T^{\frac{1}{2}-\kappa}}{1-\left\|\mathcal{L}_{g, \Psi}\right\|_{\mathcal{B}\left(\mathcal{X}_{T}^{\kappa}\left(\mathcal{C}^{\frac{1}{2}+\kappa}\right)\right)}} .
$$

This shows that $\mathcal{V}_{T}$ is continuous on $A_{M}$ when $T<T_{0}(M, M)$.

Now suppose (as an inductive hypothesis) that $\mathcal{V}_{T}$ is continuous on $A_{M}$ and let $T^{\prime} \in$ $\left(T, T+T_{0}(M, M)\right)$. For $(g, \Psi, \underline{v}),(\tilde{g}, \tilde{\Psi}, \underline{\tilde{v}}) \in A_{M}$, put $v=\mathcal{V}_{T}(g, \Psi, \underline{v})$ and $\tilde{v}=\mathcal{V}_{T}(\tilde{g}, \tilde{\Psi}, \underline{\tilde{v}})$. For $t \in\left[T, T^{\prime}\right]$, let $w(t, x)=v(t-T, x)$ and let $\tilde{w}(t, x)=\tilde{v}(t-T, x)$. We have $w=$ $\mathcal{V}_{T^{\prime}-T}(g, \Psi, v(T, \cdot))$ and $\tilde{w}=\mathcal{V}_{T^{\prime}-T}(g, \Psi, \tilde{v}(T, \cdot))$. Since $\mathcal{V}_{T^{\prime}-T}$ is continuous, so is $\mathcal{V}_{T^{\prime}}$.

By induction, this implies that $\mathcal{V}_{T}$ is continuous on $A_{M}$ for any $T$. Since this is true for any $M$, we see that, for any $T, \mathcal{V}_{T}$ is in fact continuous on $\mathcal{C}^{-\kappa} \times \mathcal{B}\left(\mathcal{C}^{\frac{1}{2}+\kappa}, \mathcal{C}^{-\kappa}\right) \times \mathcal{C}^{-\frac{1}{2}+2 \kappa}$.

The continuity of the solution map then allows us to apply the stability results proved in Section 5 to show that the solutions converge.

Corollary 6.5. If $\underline{u} \in \mathcal{C}^{-\frac{1}{2}+2 \kappa}$, then for all $\varepsilon \in[0,1)$, for any $T>0$ there is a unique solution $v_{\varepsilon} \in \mathcal{X}_{T}^{\kappa}\left(\mathcal{C}^{\frac{1}{2}+\kappa}\right)$ to (3.1), and $v_{\varepsilon}$ converges to $v_{0}$ in probability in $\mathcal{X}_{T}^{\kappa}\left(\mathcal{C}^{\frac{1}{2}+\kappa}\right)$.

Proof. As noted above, $v_{\varepsilon}=\mathcal{V}_{T}\left(Z_{\varepsilon}-F * \xi_{\varepsilon}, \Xi_{\varepsilon}, \mathrm{e}^{-S_{\varepsilon}} \underline{u}\right)$ uniquely solves (3.1). Using (2.4), the periodicity of $\xi$ and $\xi_{\varepsilon}$, and Lemma 5.1, we see that $F * \xi_{\varepsilon} \rightarrow F * \xi$ as $\varepsilon \rightarrow 0$ in probability in $L^{\infty}$. Proposition 5.3 says that $Z_{\varepsilon} \rightarrow Z_{0}$ in probability in $\mathcal{C}^{-\kappa}$, and Proposition 5.11 says that $\Xi_{\varepsilon} \rightarrow \Xi_{0}$ in probability in $\mathcal{B}\left(\mathcal{C}^{\frac{1}{2}+\kappa}, \mathcal{C}^{-\kappa}\right)$. Also, Lemmas 5.2 and 2.2, along with the assumption $\underline{u} \in \mathcal{C}^{-\frac{1}{2}+2 \kappa}$, imply that $\mathrm{e}^{-S_{\varepsilon}} \underline{u} \rightarrow \mathrm{e}^{-S_{0}} \underline{u}$ in probability in $\mathcal{C}^{-\frac{1}{2}+2 \kappa}$. Thus Proposition 6.4 implies that $v_{\varepsilon} \rightarrow v_{0}$ in probability in $\mathcal{X}_{T}^{\kappa}\left(\mathcal{C}^{\frac{1}{2}+\kappa}\right)$.

To prove Theorem 1.1, it simply remains to undo the change of variables.

Proof of Theorem 1.1. For $\varepsilon>0$, we have $u_{\varepsilon}=\mathrm{e}^{S_{\varepsilon}} v_{\varepsilon}$ by Lemma 3.1. Lemma 5.2, Corollary 6.5, and Lemma 2.2 imply that as $\varepsilon \downarrow 0, u_{\varepsilon}$ converges in probability to $\mathrm{e}^{S_{0}} v_{0}$ in $\mathcal{X}_{T}^{\kappa}\left(\mathcal{C}^{\frac{1}{2}-\kappa}\right)$. The estimate (1.4) for $C_{\varepsilon}$ was proved as Proposition 5.9.

\section{References}

[1] Hajer Bahouri, Jean-Yves Chemin, and Raphael Danchin. Fourier analysis and nonlinear partial differential equations, volume 343 of Grundlehren Math. Wiss. Springer, Heidelberg, 2011. MR-2768550

[2] Nils Berglund and Christian Kuehn. Model spaces of regularity structures for space-fractional SPDEs. J. Stat. Phys., 168(2):331-368, 2017. MR-3667364

[3] L. Chiarini and C. Landim. A one-dimensional non-local singular SPDE, December 2019. arXiv:1912.11869v1 [math.PR].

[4] Arnaud Debussche and Jorg Martin. Solution to the stochastic Schrodinger equation on the full space. Nonlinearity, 32(4):1147-1174, 2019. MR-3923163 
[5] Arnaud Debussche and Hendrik Weber. The Schrodinger equation with spatial white noise potential. Electron. J. Probab., 23:Paper No. 28, 2018. MR-3785398

[6] Marco Furlan and Jean-Christophe Mourrat. A tightness criterion for random fields, with application to the Ising model. Electron. J. Probab., 22:Paper No. 97, 2017. MR-3724565

[7] $\mathrm{Yu} \mathrm{Gu}$ and Jingyu Huang. Chaos expansion of 2D parabolic Anderson model. Electron. Commun. Probab., 23:Paper No. 26, 2018. MR-3798237

[8] Yu Gu and Weijun Xu. Moments of 2D parabolic Anderson model. Asymptot. Anal., 108(3):151161, 2018. MR-3817383

[9] Massimiliano Gubinelli, Peter Imkeller, and Nicolas Perkowski. Paracontrolled distributions and singular PDEs. Forum Math. Pi, 3:e6, 2015. MR-3406823

[10] Martin Hairer. A theory of regularity structures. Invent. Math., 198(2):269-504, 2014. MR3274562

[11] Martin Hairer and Cyril Labbe. A simple construction of the continuum parabolic Anderson model on $\mathbf{R}^{2}$. Electron. Commun. Probab., 20(43):1-11, 2015. MR-3358965

[12] Martin Hairer and Cyril Labbe. Multiplicative stochastic heat equations on the whole space. J. Eur. Math. Soc., 20(4):1005-1054, 2018. MR-3779690

[13] Wolfgang Konig. The parabolic Anderson model. Random walk in random potential. Pathw. Math. Birkhauser/Springer, 2016. MR-3526112

[14] Mateusz Kwasnicki. Ten equivalent definitions of the fractional Laplace operator. Fract. Calc. Appl. Anal., 20(1):7-51, 2017. MR-3613319

[15] Stanislav Molchanov and Hao Zhang. The parabolic Anderson model with long range basic Hamiltonian and Weibull type random potential. In Probability in complex physical systems. Volume 11, Springer Proc. Math., pp. 13-31. Springer, Heidelberg, 2012. MR-3372843

Acknowledgments. We thank Lenya Ryzhik for suggesting the problem and much useful advice, as well as Yu Gu, Leonid Mytnik, and Weijun Xu for interesting conversations. We are also grateful to Leandro Chiarini for pointing out a subtlety in the proof of Lemma 2.5. We thank an anonymous referee for a very careful reading of the manuscript and several important comments. The author was partially supported by the NSF Graduate Research Fellowship Program under Grant No. DGE-1147470. 